\title{
RADIO-ISOTOPE PRODUCTION USING LASER WAKEFIELD ACCELERATORS
}

\author{
W.P. Leemans, D. Rodgers, P.E. Catravas, C.G.R. Geddes, G. Fubiani, C. Toth, \\ E. Esarey, B.A. Shadwick, R. Donahue, A. Smith, LBNL, Berkeley, CA, USA *
}

A. Reitsma, Technische Universiteit Eindhoven, the Netherlands

\begin{abstract}
A $10 \mathrm{~Hz}, 10 \mathrm{TW}$ solid state laser system has been used to produce electron beams suitable for radio-isotope production. The laser beam was focused using a $30 \mathrm{~cm}$ focal length $\mathrm{f} / 6$ off-axis parabola on a gas plume produced by a high pressure pulsed gas jet. Electrons were trapped and accelerated by high gradient wakefields excited in the ionized gas through the self-modulated laser wakefield instability. The electron beam was measured to contain in excess of $5 \mathrm{nC} / \mathrm{bunch}$. A composite $\mathrm{Pb} / \mathrm{Cu}$ target was used to convert the electron beam into $\gamma$-rays which subsequently produced radio-isotopes through $(\gamma, \mathrm{n})$ reactions. Isotope identification through $\gamma$-ray spectroscopy and half-life time measurements demonstrated that ${ }^{61} \mathrm{Cu}$ was produced which indicates that $20-25 \mathrm{MeV} \gamma$-rays were produced, and hence electrons with energies greater than $25-30 \mathrm{MeV}$. The production of high energy electrons was independently confirmed using a bending magnet spectrometer. The measured spectra had an exponential distribution with a $3 \mathrm{MeV}$ width. The amount of activation was on the order of 2.5 $\mu \mathrm{Ci}$ after 3 hours of operation at $1 \mathrm{~Hz}$. Future experiments will aim at increasing this yield by post-accelerating the electron beam using a channel guided laser wakefield accelerator.
\end{abstract}

\section{INTRODUCTION}

We discuss measurements of nuclear activation in lead and copper targets using electron beams produced by a self modulated-laser wakefield accelerator (SM-LWFA) [1] driven by the $10 \mathrm{TW}, 10 \mathrm{~Hz}$ l'OASIS laser [1-3]. In this SM-LWFA regime [4-10] a single, long laser pulse with duration $L>\lambda_{p}$ breaks up into a train of short pulses, each of which has a width on the order of the plasma wavelength $\lambda_{p}$. Associated with the break up of the long pulse and the formation of the pulse train is a large amplitude plasma wakefield. The fields of this wave are sufficiently large to self-trap electrons from the background plasma, and accelerate them to high energies $(\approx 100 \mathrm{MeV})$.

On-line detection of neutron and gamma radiation was used to optimize the performance of the laser driven accelerator. Details of the experiment are presented followed by a discussion on how to increase the activation yield.

\footnotetext{
${ }^{*}$ Work supported by Department of Energy under Contract No. DEAC-03-76SF0098; C.G.R.G. acknowledges the Hertz Foundation for support.
}

\section{$2 \gamma$-NEUTRON ACTIVATION: PROOF-OF-PRINCIPLE EXPERIMENTS}

$\gamma, \mathrm{n}$ activation relies on stopping an electron beam in an appropriate target and using the bremsstrahlung $\gamma$-rays to activate the target material. The reaction products are then analyzed by $\gamma$-spectroscopy for identification. The various reactions, $(\gamma, \mathrm{n}),(\gamma, 2 \mathrm{n})$, and $(\gamma, 3 \mathrm{n})$, have $\gamma$-ray threshold energies that must be exceeded in order for the reaction to occur. These thresholds generally increase by $10-15 \mathrm{MeV}$ per neutron released in a given reaction.

\subsection{Experimental Set-Up and Results}

The lay-out of the experiment is shown in Fig. 1. The high power laser beam was produced with a Ti: $\mathrm{Al}_{2} \mathrm{O}_{3}$ laser which is described in Ref. [1-3].

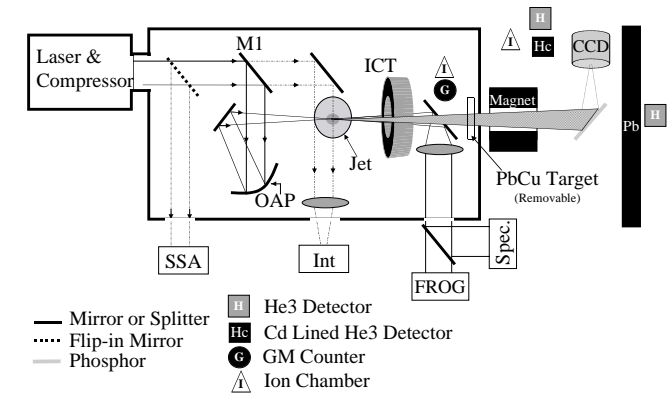

Figure 1: Lay-out of experiment showing the laser beam exiting the compressor, being reflected by mirror M1 onto the off-axis parabola (OAP), which focuses it onto the gasjet. The resulting electron beam is measured using the integrated current transformer (ICT) and is dispersed in the magnetic spectrometer onto a phosphor screen. The screen is imaged with the CCD. Plasma densities are measured with the interferometer (INT) and the laser beam is analyzed using the single-shot autocorrelator (SSA), the frequency resolved optical gating system (FROG) and an imaging optical spectrometer (Spec.).

High energy (up to $1 \mathrm{~J}$ per pulse), 200-300 ps duration chirped laser pulses were propagated into the l'OASIS shielded cave below the laser lab through an evacuated beam pipe and compressed in a vacuum compressor to peak powers of 8-10 TW in pulses as short as $50 \mathrm{fs}$. After compression, the laser beam was reflected with mirror M1 onto an $\mathrm{F} / 4,30 \mathrm{~cm}$ focal length off-axis parabola (OAP), which focused the beam $(w=6 \mu \mathrm{m})$ onto a high pressure pulsed 
gasjet. The gasjet was operated with $\mathrm{H}_{2}, \mathrm{He}$ and $\mathrm{N}_{2}$ at backing pressures up to 72 bar. A final steering mirror after the OAP was used to provide independent control of the pointing direction. After the interaction region, the main laser beam was reflected by a gold or silver coated, $2 \mu \mathrm{m}$ thick nitrocellulose pellicle. This material and thickness was chosen to minimize Coulomb scattering of electrons propagating through it, while still maintaining optical flatness.

The typical plasma density profiles were parabolic with a diameter (FWHM) around $1.2 \mathrm{~mm}$ and had peak densities on the order of $n_{e}=1-3 \times 10^{19} \mathrm{~cm}^{-3}$. The laser peak power was varied using both the pulse duration and laser energy. For the results discussed here the laser peak power reached $8.3 \mathrm{TW}$. The total charge per bunch of the electron beam was measured using a commercial integrating current transformer (ICT). The spatial profile was measured with a phosphor screen that was imaged onto a 16 bit charge coupled device (CCD) camera. The energy distribution of the electron beam was measured with a dipole spectrometer magnet.

Neutron and gamma rays were monitored with a variety of detectors, allowing both use of this radiation as a beam diagnostic and for the evaluation of various detector performances.

\subsection{Electron, neutron and $\gamma$-ray correlations.}

In Fig. 2, electron, neutron and $\gamma$-ray yield (measured near the target) versus position of the gasjet is shown. Here the zero position refers to the vacuum laser focus position coinciding with the center of the gasjet. In general, the

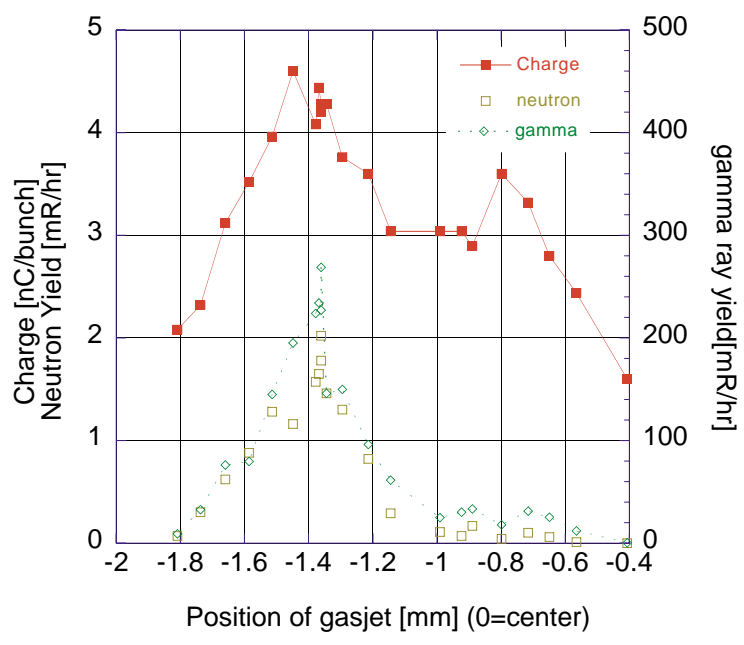

Figure 2: Electron and neutron yield versus gas jet position. The center of the gasjet is at $0 \mathrm{~mm}$. The first peak in electron yield at $\sim 1.4 \mathrm{~mm}$ correlates with neutron and $\gamma$-ray yield indicating that a significant amount of high energy electrons is produced. The absence of a neutron or $\gamma$-ray peak near the second peak in electron yield at $\sim 0.7 \mathrm{~mm}$ is indicative of lower energy electrons being generated. different yields were found to be very well correlated with each other and large increases in yield were observed by adjusting the position of the gasjet front edge with respect to the location of the vacuum focus. The neutron yield was also found to increase with forward directed $\gamma$-ray yield, consistent with the production of higher energy, more collimated electrons. As can be seen in Fig. 2, electron, neutron and $\gamma$-ray yields peaked for a gas jet position around $\sim 1.4 \mathrm{~mm}$. In this position the laser beam is focused on the front edge, providing for the longest interaction distance between the high intensity laser pulse and the gasjet. This in turn can result in the longest acceleration distance and hence highest energy gain for trapped electrons. Also, due to the parabolic density profile of the gasjet, dephasing length of the electrons may be increased due to plasma waves moving up a density gradient.

A second maximum for the electron yield was obtained for a gasjet position around $\sim 0.8 \mathrm{~mm}$. However, neutron and $\gamma$-ray yield near the target were considerably lower indicating that electrons produced at this gasjet position have lower energy. We hypothesis that, for laser pulses focused into the gasjet plume, ionization induced refraction effects [11] could prevent the laser pulse from reaching the vacuum spot size leading to a reduced peak intensity and hence lower amplitude of the excited laser wakefield. In addition, the net interaction distance could be reduced due to the fact that less plasma is ahead of the laser pulse when it is focused inside the jet, leading to a further reduction of the net energy gain.

The yield in electrons and neutrons was also found to scale with increased laser power. The minimum power at which neutrons were observed was on the order of $2.8 \mathrm{TW}$, which is comparable to the critical power for $n_{e}=10^{19}$ $\mathrm{cm}^{-3}$. In addition, the neutron yield was found to be dependent on laser chirp, i.e., significantly larger yields were obtained for positively chirped laser pulses (red wavelengths in the front of the pulse, blue in the back). Several possible mechanisms for this effect are being investigated at the present time.

\subsection{Gamma-ray Analysis of the Target}

After approximately 3 hours of target activation, the target was removed from the vacuum chamber and prepared for counting. Three intrinsic Germanium $\gamma$-ray spectrometers were used, with the firsts counts beginning within 15minutes of the beam-off time. The vast majority of activity seen in the first minutes of counting was at a $\gamma$-ray energy of $511 \mathrm{keV}$, as expected. The activation decay followed a 10-minute half-life at first, indicating that significant ${ }^{62} \mathrm{Cu}$ was in the sample, produced from a $(\gamma, \mathrm{n})$ reaction on ${ }^{63} \mathrm{Cu}$, which has a $10.8 \mathrm{MeV}$ threshold. From the initial 511$\mathrm{keV}$ decay, we resolved the 9.7-minute half-life of the ${ }^{62} \mathrm{Cu}$ from the ${ }^{63} \mathrm{Cu}(\gamma, \mathrm{n})$ reaction (10.8 MeV threshold) and used this to derive the ${ }^{62} \mathrm{Cu}$ activity at the end of the run. Successful observation of the $3.3 \mathrm{hr}$ half-life time of ${ }^{61} \mathrm{Cu}$ from the ${ }^{63} \mathrm{Cu}(\gamma, 2 \mathrm{n})$ reaction confirmed that a significant frac- 
tion of the $\gamma$-ray flux, and hence electron beam, had an energy above the $19.7 \mathrm{MeV}$ threshold for this reaction. Using a Monte-Carlo simulation based code, an estimate was made of the induced activity of ${ }^{62} \mathrm{Cu}$, based on the measured electron distribution. The simulated activation was found to be $2.12 \mu \mathrm{Ci}$ compared to $2.5 \mu \mathrm{Ci}$ in the experiment.

\section{ACTIVATION YIELD ENHANCEMENT USING DENSITY CHANNELS}

The activity generated in these experiments was on the order of $2.5 \mu \mathrm{Ci}$. To become competitive with conventional cyclotrons would require 4-5 orders of magnitude increase in activation. The most straightforward approach is increasing the repetition rate of the system. Our initial experiments were operating typically at $1-3 \mathrm{~Hz}$, limited by the gas load on the vacuum system. We have recently upgraded our vacuum pumping system to allow $10 \mathrm{~Hz}$ operation, which is the maximum repetition rate of the laser system. Multi-terawatt systems are being developed around the world that can operate at $100-1000 \mathrm{~Hz}$ with average power reaching $100 \mathrm{~W}$. Since the activation is proportional to the number of $\gamma$-ray photons (i.e., electrons) at the "resonant" energy, an alternative way to increase the activation is to increase the beam energy. This can be achieved by (1) increasing the laser power and (2) post-accelerating the beam to increase the amount of electrons at the optimum energy. Here we discuss the post-acceleration approach.

The amount of background plasma electrons captured and accelerated in a SMLWFA can be extremely large (multi-nC per bunch) and well collimated bright beams emerge from the plasma. The mean energy is typically less than $10 \mathrm{MeV}$ with $100 \%$ energy spread. The mean electron energy can be greatly enhanced, however, by injecting the electron beam emerging from the SMLWFA into a channelguided LWFA (CGLWFA). The CGLWFA is a LWFA that operates in the standard regime (laser pulse length approximately equal to the plasma wavelength, $L \simeq \lambda_{p}$ ) that relies on channel guiding to prevent pulse diffraction and extend the acceleration distance. Simulations were done using a fully non-linear, relativistic hydrodynamic description for the plasma wave, combined with test particle methods for the description of the bunch, including the effects of beam loading [12].

In the simulations, a bunch of electrons containing 45 $\mathrm{pC}$, with an energy spread given by the experimentally measured distribution, was injected into a laser excited plasma channel. The channel width (peak-to-peak) was 45 $\mu \mathrm{m}$ with an on-axis density of $10^{18} \mathrm{~cm}^{-3}$. The laser power was $1.7 \mathrm{TW}$ focused to a spot size of $8 \mu \mathrm{m}$. A large fraction of the initial low energy electrons gained energy in excess of $30 \mathrm{MeV}$ after propagating $2 \mathrm{~mm}$. The electron energy distributions (a) initially, and after the beam propagates (b) $2 \mathrm{~mm}$ and (c) $5 \mathrm{~mm}$ in the channel, are shown in Fig. 3. The fraction of electrons with energy above $25 \mathrm{MeV}$ was increased from $0.012 \%$ to more than $24 \%$, representing an increase in yield of more than three orders of magnitude. Further optimization studies, including PIC code runs, will

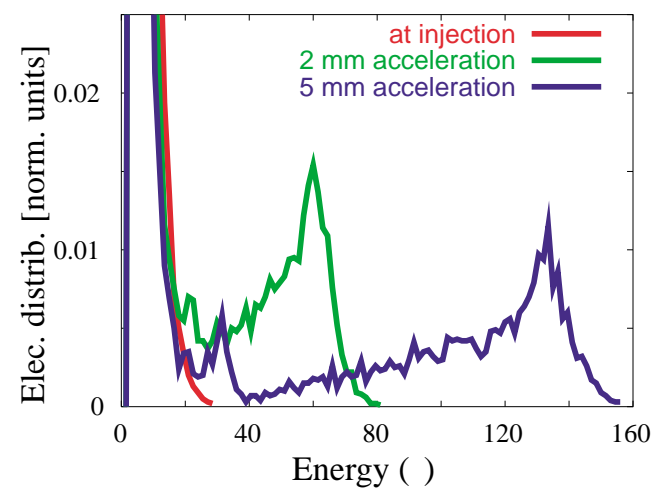

Figure 3: Energy distribution of electrons at injection (red curve) and after propagating $2 \mathrm{~mm}$ (green curve) and $5 \mathrm{~mm}$ (blue curve) in the plasma channel.

be carried out to optimize the fraction of accelerated electrons.

\section{REFERENCES}

[1] W. P. Leemans, D. Rodgers, P. Catravas, C. Geddes, G. Fubiani, E. Esarey, B. Shadwick, R. Donahue, and A. Smith, Phys. Plasmas 8, 2510 (2001).

[2] W. P. Leemans, P. Volfbeyn, K. Z. Guo, S. Chattopadhyay, C. B. Schroeder, B. A. Shadwick, P. B. Lee, J. S. Wurtele, and E. Esarey, Phys. Plasmas 5, 1615 (1998).

[3] P. Volfbeyn, E. Esarey, and W. Leemans, Phys. Plasmas 6, 2269 (1999).

[4] P. Sprangle, E. Esarey, J. Krall, and G. Joyce, Phys. Rev. Lett. 69, 2200 (1992).

[5] E. Esarey, P. Sprangle, J. Krall, and A. Ting, IEEE Trans. Plasma Sci. 24, 252 (1996).

[6] W. B. Mori, C. D. Decker, D. E. Hinkel, and T. Katsouleas, Phys. Rev. Lett. 72, 1482 (1994).

[7] A. Modena, Z. Najmudin, A. E. Dangor, C. E. Clayton, K. A. Marsh, C. Joshi, V. Malka, C. B. Darrow, C. Danson, D. Neely, and F. N. Walsh, Nature 377, 606 (1995).

[8] K. Nakajima, D. Fisher, T. Kawakubo, H. Nakanishi, A. Ogata, Y. Kato, Y. Kitagawa, R. Kodama, K. Mima, H. Shiraga, K. Suzuki, K. Yamakawa, T. Zhang, Y. Sakawa, T. Shoji, Y. Nishida, N. Yugami, M. Downer, and T. Tajima, Phys. Rev. Lett. 74, 4428 (1995).

[9] D. Umstadter, S.-Y. Chen, A. Maksimchuk, G. Mourou, and R. Wagner, Science 273, 472 (1996).

[10] A. Ting, C. I. Moore, K. Krushelnick, C. Manka, E. Esarey, P. Sprangle, R. Hubbard, H. R. Burris, and M. Baine, Phys. Plasmas 4, 1889 (1997).

[11] W. P. Leemans, C. E. Clayton, W. B. Mori, K. A. Marsh, P. K. Kaw, A. Dyson, C. Joshi, and J. M. Wallace, Phys. Rev. A 46, 1091 (1992).

[12] A. J. W. Reitsma, V. V. Goloviznin, L. P. J. Kamp, and T. J. Schep, Phys. Rev. E 63, 1 (2001). 